Article

\title{
The Role of the Bacterial Community in Producing a Peculiar Smell in Chinese Fermented Sour Soup
}

\author{
Liangjing Lin ${ }^{1}$, Jinyuan $\mathrm{Wu}^{1}{ }^{1}, \mathrm{Xi}$ Chen ${ }^{1}$, Libiao Huang ${ }^{1}$, Xiaoyong Zhang ${ }^{2, *}$ and \\ Xiangyang Gao ${ }^{1, *}$ \\ 1 Guangdong Provincial Key Laboratory of Nutraceuticals and Functional Foods, College of Food Science, \\ South China Agricultural University, Guangzhou 510642, China; jing109@stu.scau.edu.cn (L.L.); \\ wjy1713263483@163.com (J.W.); cxjenny123@163.com (X.C.); 15768309718@163.com (L.H.) \\ 2 Joint Laboratory of Guangdong Province and Hong Kong Region on Marine Bioresource Conservation and \\ Exploitation, College of Marine Sciences, South China Agricultural University, Guangzhou 510642, China \\ * Correspondence: zhangxiaoyong@scau.edu.cn (X.Z.); gaoxiangyang@scau.edu.cn (X.G.); \\ Tel.: +86-138-2840-3139 (X.Z.); +86-136-6008-9801 (X.G.)
}

Received: 29 June 2020; Accepted: 11 August 2020; Published: 21 August 2020

\begin{abstract}
In this paper, the volatile flavour constituents and the bacterial diversity in characteristic Chinese fermented sour soup were analysed, and the dynamics of bacteria associated with the odour were characterized. The bacterial diversity of sour soup was studied by high-throughput sequencing. A total of 10 phyla and 89 genera were detected. Firmicutes was the dominant phylum of sour soup, accounting for $87.14-98.57 \%$. The genus structure of normal sour soup was relatively simple, and Lactobacillus (78.05-90.26\%) was the dominant genus. In addition to Lactobacillus, the foul-smelling sour soup contained more Pediococcus spp., Caproiciproducens spp., and Clostridium-sensu-stricto $12 \mathrm{spp}$. (relative abundance $>1 \%$ ) than the normal sour soup. A total of 51 aroma compounds were detected by gas chromatography-mass spectrometry(GC-IMS), including 25 esters, 8 terpenes, 8 alcohols, 3 sulfur compounds, 2 acids, 2 ketones, 1 pyrazine, 1 monoterpene and 1 aldehyde. According to the relative odour active value (ROAV) calculation, 51 important flavour-contributing substances and 7 flavour-coordinating substances were determined. The esters with the highest relative percentages and ROAV values provided the pleasant flavour of the sour soup. In the foul-smelling sour soup, the ROAV values of 1,8-cineole, isobutyl acetate, ethyl butanoate, ethyl octanoate- $\mathrm{M}$, and ethyl hexanoate-M decreased, while those of diallyl disulfide-M and diallyl disulfide-D, which were probably responsible for the foul flavour, increased. Through Pearson correlation analysis, the odour production of the foul-smelling soup was determined to be related to Pediococcus spp., Caproiciproducens spp., Clostridiumsensu_stricto_12 spp., Oscillibacter spp., Bacteroides spp., Fibaculaceae_unclassified spp., Acinetobacter spp. and Halomonas spp.
\end{abstract}

Keywords: sour soup; flavour; bacteria; Chinese fermentation; GC-IMS; high-throughput sequencing

\section{Introduction}

Sour soup is a traditional fermented condiment of Dong and Miao nationalities in Guizhou, China [1]. It is usually fermented naturally and can be made into red and white sour soup. Red sour soup is often made from ingredients such as peppers or tomatoes. Kaili Red sour soup is a typical Guizhou sour soup with a fresh, sweet, and spicy flavour. It was awarded the Guizhou Geographical Indication Protection Product in 2013. In recent years, Guizhou sour soup has gradually expanded outside of Guizhou and become one of the three characteristic hot pot ingredients, alongside Chongqing spicy hot pot sauce and Inner Mongolia clear-soup instant lamb hot pot sauce, with a huge market potential [2]. However, the total output value of Guizhou sour soup is only 240 million yuan, and 
the degree of industrialization of sour soup is relatively low. In addition, turning the paste into a cylinder during production is an important process that can result in the mixture of the salt and slurry. When the operation of turning the paste is insufficient, it will easily cause the sour soup to become stale and foul smelling. Deterioration of sour soup is a common problem in the production process and greatly affects the industrialization process of sour soup [3]. He Yangbo et al. used electronic nose and gas chromatography-mass spectrometry (GC-IMS) technology to analyse and compare the flavour differences between the foul-smelling sour soup and the normal sour soup and speculated that the foul odour of the sour soup originated from butyric acid and aldehydes [4]. Yang Jinjun et al. used an electronic nose and GC-MS to study the flavour difference of sour soup and found that the main flavours of different brands of sour soup were different [5]. Qiqi Wang et al. studied different kinds of sour soup by high-throughput sequencing and found that Lactobacillus plantarum was a common bacterium in the fermented sour soup made from different raw materials, and this bacterium played an important role in the flavour of sour soup [6]. There is a close relationship between flavour formation and the microbes involved in fermentation; however, there are few reports on the relationship between the microbes and flavour generation of sour soup, and there is also a lack of research on the origin of the foul odour of sour soup.

At present, high-throughput sequencing and GC-IMS technology have been widely used in microbial and flavour detection of fermented vegetables [7,8]. Li et al. detected the volatile flavour compounds produced during the curing of ginger and found that the content of heptanoic acid and heptanone decreased, while the content of butyral, butanone, and methionine increased, leading to a change in the flavour of pickled ginger [9]. HuipengLiang et al. used high-throughput sequencing to reveal the traditional Chinese fermentation Paocai and obtained useful information [10]. We believe that high-throughput sequencing and GC-IMS analysis may be a good means to explore the bacterial diversity and flavour variations in sour soup.

In this paper, the correlation between the bacteria and the volatile flavour components of sour soup were studied, and the bacteria that produced the foul-smelling substances in the sour soup were identified, laying the foundation for controlling the production of the odour in the sour soup and promoting the development of the sour soup industry.

\section{Materials and Methods}

\subsection{Sample Collection and Analysis}

The samples were provided by Majiang Mingyang Food Co., Ltd., located in Guizhou province, China. The sample manufacturing flow and collection was as follows: (1) Fresh tomatoes were clean and pulped. (2) The tomato pulp was fermented with salt in a fermentation tank with jar that was turned over during the fermentation period to mix the salt evenly [11]. If the salt is not mixed evenly, the sour soup can become putrid with a foul smell. The foul-smelling sour soup was collected as the sample STF, while the normal sour soup was collected as the control group (TF). The samples were sampled in sextuplicate according to the five-point sampling method studied by DU et al. [12]. A sample was prepared by mixing equal amounts of mixture from the five points: the surface, middle and bottom of the four corners and the midpoint of the fermentation tank, and collected in a sterilized sampling bottle, transported on dry ice to the laboratory and stored at $-80^{\circ} \mathrm{C}$ without loss for further analysis.

\subsection{Illumina MiSeq Sequencing}

Under aseptic conditions, $5 \mathrm{~mL}$ samples were thoroughly mixed, and a HiPure Soil DNA kit (Magen, Guangzhou China) was used to extract the total DNA of the samples. A Qubit ${ }^{\circledR}$ dsDNA HS Assay Kit (Qubit v. 3.0, Hilden Germany) was used for the determination of DNA concentration [13]. The purified DNA was used for bacterial 16S rRNA amplification. A total of 20-30 ng DNA was used as a template; the PCR primers included the "CCTACGGRRBGCASCAGKVRVGAAT" sequence and "GGACTACNVGGGTWTCTAATCC" sequence and were designed by GENEWIZ Company 
(GeneWiz, South Plainfield, NJ, USA) to amplify the V3 and V4 variable regions. In addition, an index connector was added to the end of the $16 \mathrm{~S}$ rDNA product by PCR for NGS sequencing [14]. The PCR parameters were as follows: predenaturation at $94{ }^{\circ} \mathrm{C}$ for $3 \mathrm{~min} \times 1$, denaturation at $94{ }^{\circ} \mathrm{C}$ for $5 \mathrm{~s}$, annealing at $57^{\circ} \mathrm{C}$ for $90 \mathrm{~s}$, elongation at $72{ }^{\circ} \mathrm{C}$ for $10 \mathrm{~s}$, and final elongation at $72{ }^{\circ} \mathrm{C}$ for $5 \mathrm{~min}$ for a total of 24 cycles. Amplification system $(20 \mu \mathrm{L}): 2.5 \mu \mathrm{L}$ TransStart Buffer, $2 \mu \mathrm{L}$ dNTPs, $1 \mu \mathrm{L} \times 2$ primer, $0.5 \mu \mathrm{L}$ TransStart Taq DNA, and $20 \mathrm{ng}$ DNA template; $\mathrm{ddH}_{2} \mathrm{O}$ was added to $25 \mu \mathrm{L}$. Library concentrations were detected by Qubit v. 3.0 Fluorometer (Invitrogen, Carlsbad, CA, USA). The library was quantified to $10 \mathrm{nM}$; PE250/FE300 double-end sequencing was performed according to the instructions of the Illumina MiSeq (Illumina, San Diego, CA, USA) instrument, and sequence information was read by MiSeq Control Software (MCS) [15].

\subsection{Volatile Flavour Component Analysis}

The FlavourSpec ${ }^{\circledR}$ system (Gesellschaft fur Analytische Sensorsy stemembH, Dortmond, Germany) was used to analyse the volatile flavour compounds in this study with an automatic sammer (CTC Analytics AG, Zwingen, Switzerland). The column system of FS-SE-54-CB-1, with a length of $15 \mathrm{~m}$ and an inner diameter of $0.53 \mathrm{~mm}$, provided by G.A.S. was used in this work. A $1 \mathrm{~g}$ sample was weighed and incubated in a $20 \mathrm{~mL}$ headspace glass sampling bottle at $40{ }^{\circ} \mathrm{C}$ for $15 \mathrm{~min}$. After incubation, a $500 \times$ L headspace sample was automatically injected into the sampler through a syringe heated to $85^{\circ} \mathrm{C}$. Nitrogen was sampled through the FS-SE-54-CB- 1 column. The flow rates were $2 \mathrm{~mL} / \mathrm{min}$ for $2 \mathrm{~min}, 15 \mathrm{~mL} / \mathrm{min}$ for $8 \mathrm{~min}$, and $100 \mathrm{~mL} / \mathrm{min}$ for $10 \mathrm{~min}$. Volatile compounds were characterized by GC $\times$ IMS library search software built into the NIST database and IMS database.

\subsection{Data Analysis}

VSEARCH (v. 1.9.6) was used for sequence clustering (the sequence similarity was set to $97 \%$ ). The 16S rRNA reference database used was Silva 132. Then, the species classification analysis of the OTU representative sequence was carried out using the RDP Classifier (Ribosomal Database Program) Bayesian algorithm. Based on the OTU analysis results, Shannon, Chao1, and other diversity indexes were calculated. IBM SPSS25.0 (IBM, Armonk, NY, USA) and Microsoft Excel (2016) were used for the normalized calculation and $t$-test of volatile flavour substances. IBM SPSS25.0 (IBM, Armonk, NY, USA) was used to calculate the Pearson correlation between bacteria and volatile flavour substances, and Cystoscope software v. 3.6 .0 (v. 3.6.0) was used to visualize the network.

\subsection{Nucleotide Sequence Accession Numbers}

In our study, the raw sequences were uploaded into the NCBI sequence read archive (SRA) database under accession number SRR12066355-SRR12066395.

\section{Results and Discussion}

\subsection{Sequencing and Alpha Diversity Analysis}

As shown in Table 1, a total of 421,235 DNA sequences were obtained. After filtering and rejecting unqualified sequences, 359,570 effective bacterial sequences were obtained from 12 samples. Based on a similarity of $97 \%$, the effective sequences were classified as operational taxonomic units (OTUs), and the number of bacterial OTUs on genera level was 170. The goods coverage of all samples was 1 , indicating that the sequencing depth was sufficient to reflect the microbial community contained in the samples. Alpha indexes including ACE, Chao1, Shannon, and Simpson were determined. The Shannon index represents the richness and uniformity of microbes, while the Simpson index is the comprehensive evaluation index of bacterial diversity [16]. The Ace index and Chao1 index represent colony richness; the larger the value is, the higher the richness. The Shannon and Simpson indexes refer to a positive correlation with colony diversity. According to the alpha diversity index, the diversity and richness of the foul-smelling sour soup were higher than those of the normal sour soup. 
Table 1. Sequencing data and alpha diversity index.

\begin{tabular}{ccccccccc}
\hline Sample & $\begin{array}{c}\text { Raw } \\
\text { Sequence } \\
\text { Number }\end{array}$ & $\begin{array}{c}\text { Effective } \\
\text { Sequence } \\
\text { Number }\end{array}$ & $\begin{array}{c}\text { Goods } \\
\text { Coverage }\end{array}$ & OTUs & Ace & Chao1 & Shannon & Simpson \\
\hline TF1 & 34041 & 28881 & 1 & 92 & 92.20 & 92 & 1.218 & 0.233 \\
TF2 & 46002 & 27874 & 1 & 96 & 96.00 & 96 & 1.122 & 0.208 \\
TF3 & 34180 & 33443 & 1 & 128 & 128.81 & 128.75 & 2.28 & 0.442 \\
TF4 & 32408 & 24870 & 1 & 91 & 91.00 & 91.60 & 1.18 & 0.204 \\
TF5 & 46002 & 43900 & 1 & 94 & 95.78 & 94 & 1.034 & 0.213 \\
TF6 & 34560 & 24673 & 1 & 103 & 124.74 & 104 & 2.08 & 0.235 \\
STF1 & 30267 & 28956 & 1 & 104 & 117.5 & 110.40 & 1.762 & 0.462 \\
STF2 & 30044 & 33951 & 1 & 98 & 104.2 & 95.80 & 2.481 & 0.529 \\
STF3 & 29351 & 23169 & 1 & 93 & 95.9 & 92.51 & 1.702 & 0.419 \\
STF1 & 30503 & 26910 & 1 & 116 & 121.74 & 118.5 & 1.863 & 0.479 \\
STF2 & 47201 & 36861 & 1 & 93 & 103.09 & 98.5 & 2.412 & 0.582 \\
STF3 & 26018 & 26082 & 1 & 90 & 94.31 & 94 & 1.877 & 0.492 \\
\hline
\end{tabular}

TF refers to the normal tomato fermentation sample; STF refers to the foul-smelling tomato fermentation sample;

(1-6) indicate a parallel sample of the same group sample.

\subsection{Analysis of the Difference Between Foul-Smelling and Normal Sour Soup}

As shown in Figure 1, the result of principal component analysis (PCA) indicated that the contribution rate of the first principal component was $53.36 \%$, the contribution rate of the second principal component was $34.12 \%$, and the cumulative contribution rate was $87.48 \%$. The data can basically include the main original data. The normal sample and foul-smelling sample were completely separated in the first principal component and the second principal component, and the difference was great. The foul-smelling samples were clustered. However, the normal samples TF3 and TF6 showed a great difference from other samples.

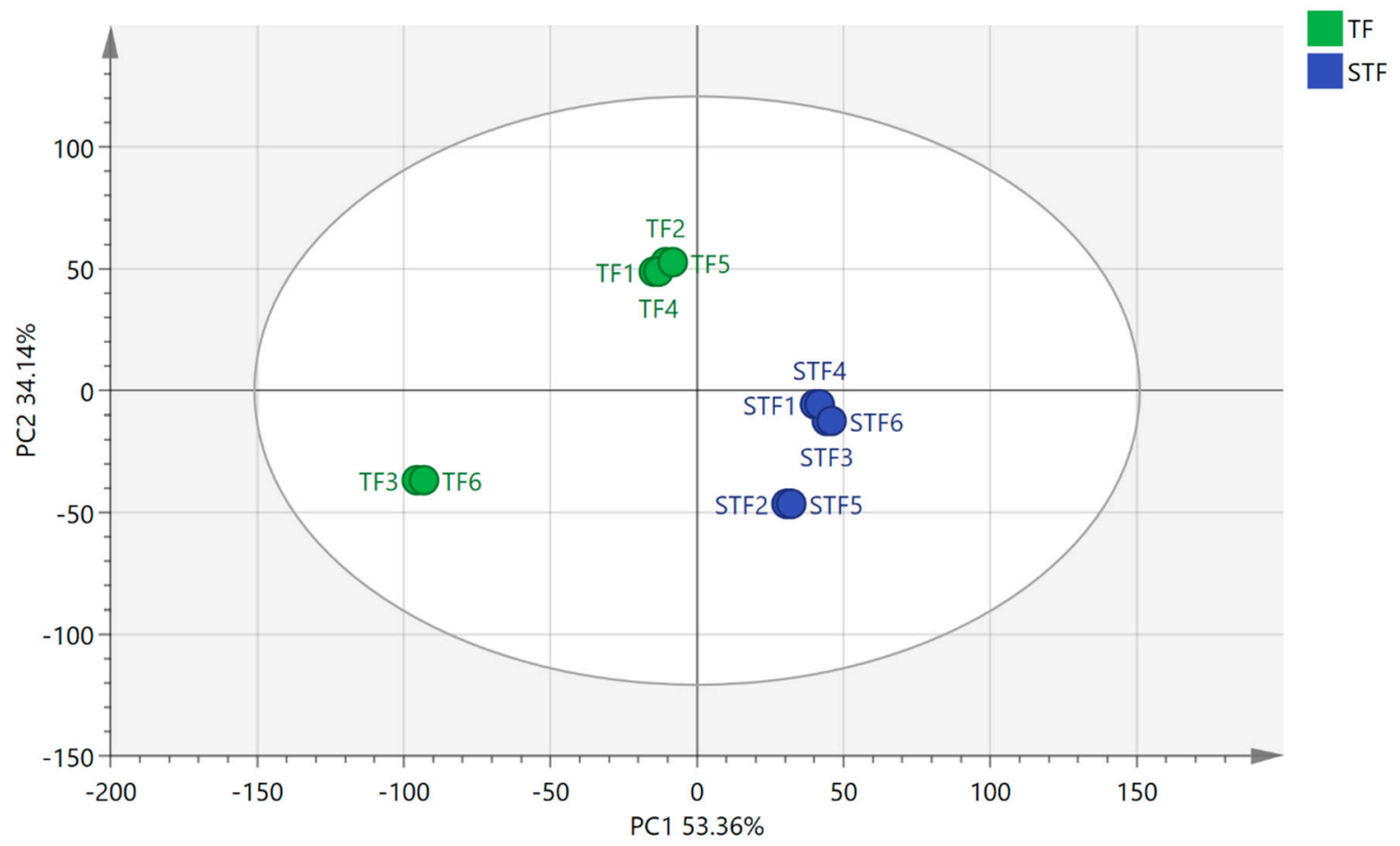

Figure 1. PCA analysis of sour soup samples. TF refers to the normal tomato fermentation sample; STF refers to the foul-smelling tomato fermentation sample; (1-6) indicate a parallel sample of the same group sample. 
A
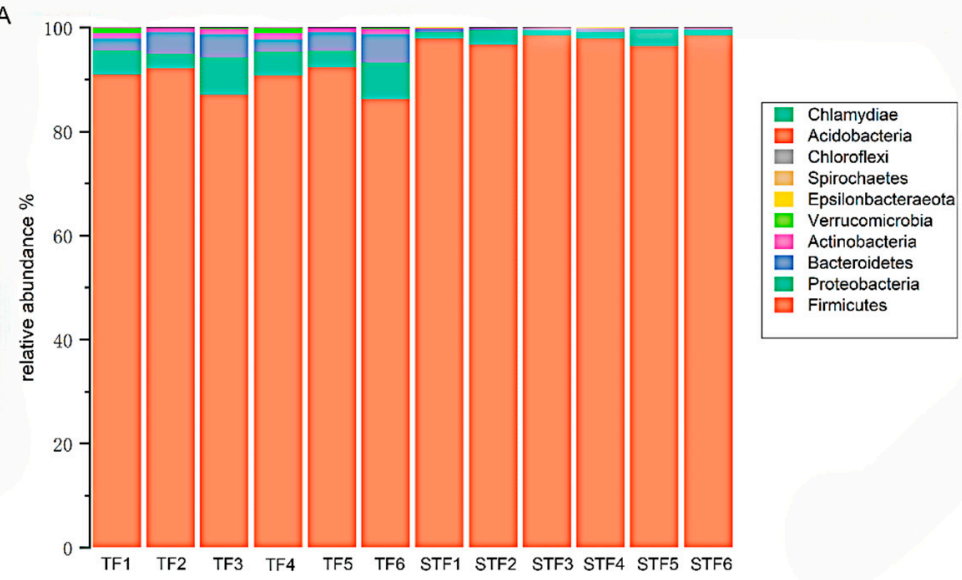

B

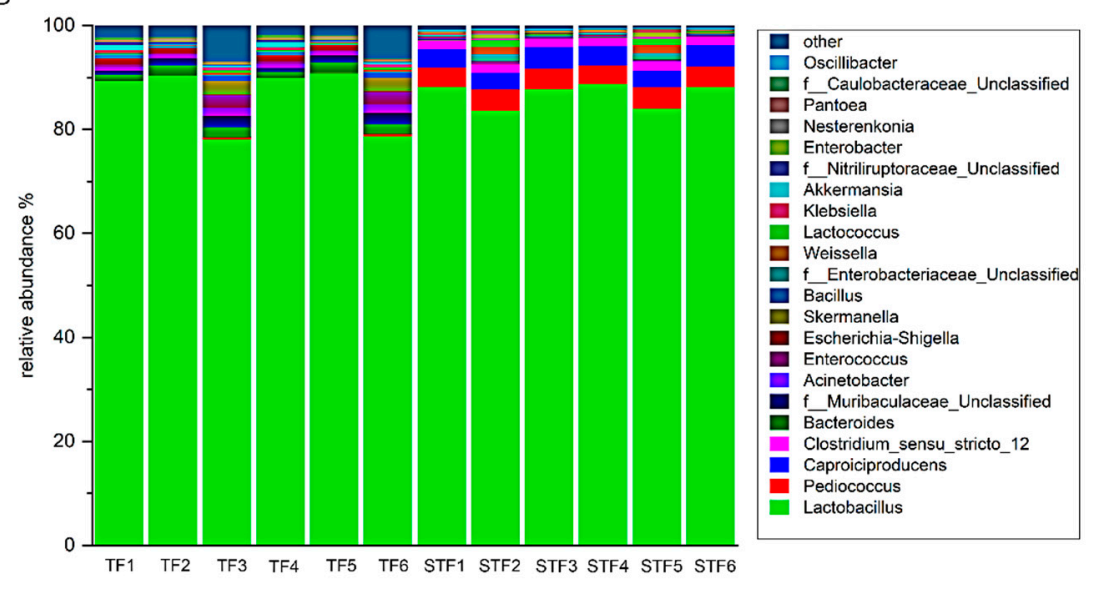

Figure 2. The relative abundance of bacteria in normal and spoilage sour soup at the phylum level (A) and genus level (B). TF refers to the normal tomato fermentation sample; STF refers to the foul-smelling tomato fermentation sample; (1-6) indicate a parallel sample of the same group sample. Each phylum or genus is represented by a unique colour. Each column represents a different studied sample.

As shown in Figure 2, a total of 10 phyla were detected. Firmicutes accounted for the largest proportion in the normal samples, accounting for approximately $89.77 \%$; firmicutes are widely found in fermented vegetables [17-19]. In addition, the relative abundances of Proteobacteria and Bacteroidetes were $5.1 \%$ and $3.65 \%$, respectively. The remaining phyla were Actinobacteria, Verrucomicrobia, Epsilonbacteraeota, Spirochaetes, Chloroflexi, Acidobacteria, and Chlamydiae, but the abundances of these phyla did not exceed $1 \%$. The main phylum of lycopene soup was similar to the studies obtained by Qiqi Wang et al., but there are slight differences in the flora with lower abundance [6]. Compared with the normal samples, the relative abundance of Firmicutes increased by $7.78 \%$ in the foul-smelling samples, accounting for $97.55 \%$. The relative abundance of Proteobacteria decreased by $3.24 \%$, accounting for $1.86 \%$. Bacteroidetes, Actinobacteria, Verrucomicrobia, and Epsilonbacteraeota were also detected at a relative abundance of less than $1 \%$. Spirochaetes, Chloroflexi, Acidobacteria, Chlamydiae, or Chlamydiae were not detected in the foul-smelling samples. At the genus level, a total of 89 genera were detected, including 80 genera in the normal samples and 54 genera in the foul-smelling samples. The top genera with the highest abundance are shown in Figure 2, while the remaining genera were classified as others. Lactobacillus $(85.88 \%)$ was the main genus in the normal samples. Lactobacillus is mainly obtained during vegetable fermentation because it 
is attached to the surface of vegetables. During the fermentation process, Lactobacillus produces a large number of organic acids, which can not only give fermented vegetables a soft sour taste and aroma but also improve nutritional value and inhibit the growth of harmful bacteria [20]. Moreover, Bacteroides spp., Muribaculaceae_Unclassified spp., and Acinetobacter spp. had relative abundances of $1.62 \%, 1.49 \%$, and $1.08 \%$, respectively. The relative abundance of other genera was not more than $1 \%$. Similarly, Lactobacillus was predominant in the foul-smelling samples, with a relative abundance of $86.52 \%$. However, compared with the normal samples, the relative abundance of other lactic acid bacteria-Pediococcus spp.and Weissella spp.-increased in the foul-smelling samples. In addition, Caproiciproducens spp.and Clostridium-sensu-stricto-12 spp., which were not found in the normal samples, had a high abundance in the foul-smelling samples, accounting for $3.67 \%$ and $1.68 \%$, respectively. Caproiciproducens spp.and Clostridium-sensu-stricto-12 spp. often appear in the fermentation of wine and can produce butyric acid and caproic acid, giving the wine a pungent and rancid creaminess [21-24].

\subsection{Analysis of the Characteristic Bacterial Flora of Normal and Foul-Smelling Sour Soup}

Linear discriminant analysis (LEfSe) analysis (Figure 3) showed that there was a significant difference in the bacterial community between the normal samples and foul-smelling samples. The relative abundance of Bacteroidetes, Bacteroidia, Bacteroidia, Bacteroidaceae, Bacteroides, Muribaculaceae, Alphaproteobacteria, and Pseudomonadales in the normal samples was significantly higher than that in the foul-smelling samples. Sensdium-stricto-12, Pediococcus, Caproiciproducens, Ruminococcaceae, Clostridiales, Clostridia and Firmicutes were higher in the foul-smelling samples than in the normal samples.

Figure 4 shows the hierarchical relationship from class to genus. The node size corresponds to the relative abundance of taxa. In the normal samples, the relatively abundant classes are Bacteroidia and Alphaproteobacteria. The orders with higher relative abundance were Bacteroidales and Pseudomonadales, while the families with higher relative abundance were Bacteroidaceae and Muribaculaceae. In the abnormal samples, the class with relatively high abundance was Clostridia, the order with a relatively high abundance was Clostridiales, and the families with relatively high abundances were Clostridiaceae and Ruminococcaceae.

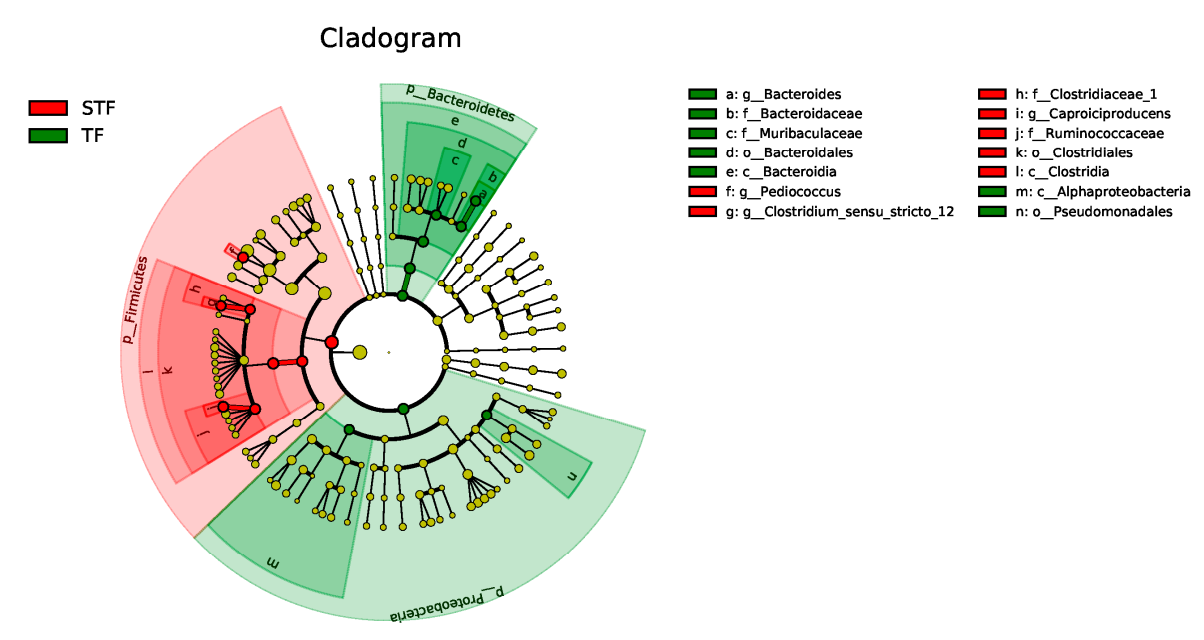

Figure 3. Cladogram of bacterial colonies in sour soup. TF refers to the normal tomato fermentation sample; STF refers to the foul-smelling tomato fermentation sample. The different colours represent the different samples. Node size corresponds to the relative abundance of taxa. 


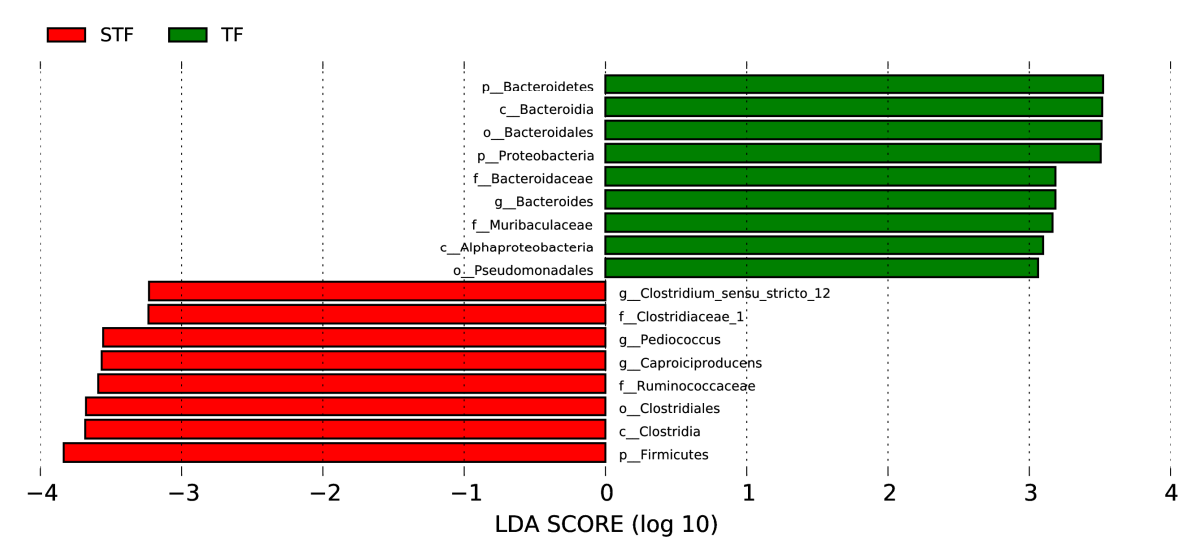

Figure 4. Trilinear discriminant analysis. TF refers to the normal tomato fermentation sample; STF refers to the foul-smelling tomato fermentation sample. The different colours represent the characteristic microorganisms of different samples. Each column represents a different microorganism.

\subsection{The Relative Content Analysis of Normal and Foul-Smelling Sour Soup}

Figure 5 (retention time, migration time, and peak strength) shows that there was a difference in volatile organic compounds between the normal samples and foul-smelling samples.

Figure 5 shows that the headspace components of the normal samples and foul-smelling samples were effectively separated, the flavour components were separated within $1000 \mathrm{~s}$, and the migration time was 7.82-7.85 ms. The signal peak was selected and marked on the ion migration spectrum, and component analysis was carried out by Library Search software built into the GC-IMS. Finally, 51 volatile components and 16 unknown components were clearly identified. The relative percentage content was calculated by the area normalization method and is summarized in Table 2 . The $t$-test analysis results of volatile components in the normal and foul-smelling samples are listed in Table 2.

Alcohols (7.44-12.17\%) were second only to esters in sour soup. They are produced by oxidative decomposition of fats or reduction in carbonyl compounds. The presence of alcohol compounds usually produces sweet, fruity, alcoholic, balsam, and green flavours and sensations. The higher content of 1-propanol in alcohols results in a floral, fruity, and grass aroma [28], while the higher content of ethanol, which is mainly produced by lactose fermentation and the catabolic metabolism of alanine, plays an important role in the formation of esters [29]. Compared with the normal samples, the relative content of ethanol and 1-propanol in the foul-smelling samples decreased.

Although only two kinds of ketone substances were detected, the relative percentage content was relatively large and generally presented a floral aroma, with stable and lasting properties. Compared with normal samples, the relative percentages of 2-butanone and acetone in the foul-smelling samples were significantly decreased. Aldehydes are mainly generated by the oxidation of unsaturated fatty acids [30]. The relative normal percentage of 3-methylbutanal in the foul-smelling soup was higher than that in the soup, which has a malty (pungent) taste that is unpleasant at high concentrations but becomes fruity and pleasant at low concentrations [31]. 


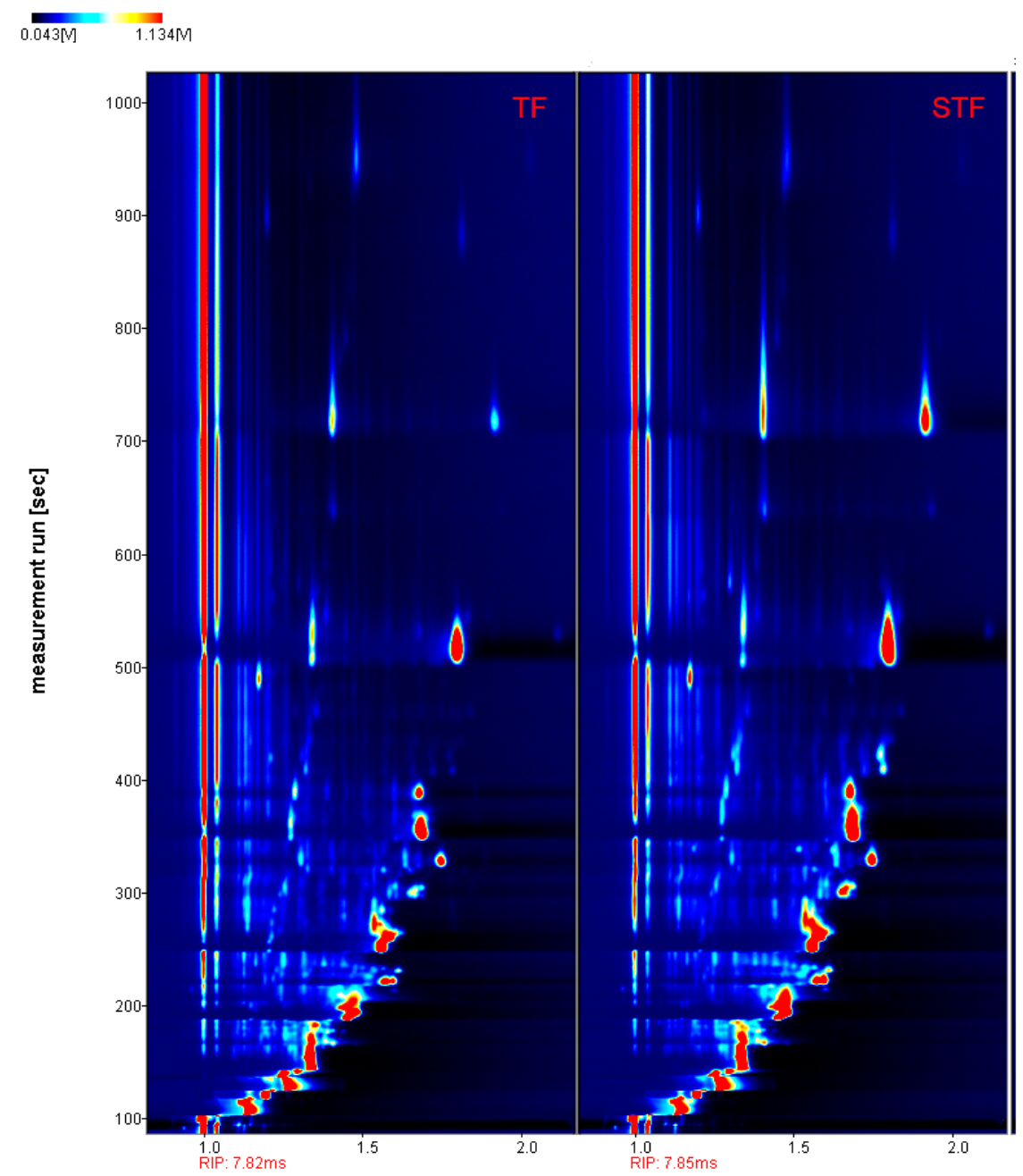

Figure 5. GC-IMS spectra of acid soup (planform). TF refers to the normal tomato fermentation sample; STF refers to the foul-smelling tomato fermentation sample. The red vertical line represents the reactive ion peak (RIP), and each point on both sides of the RIP represents a volatile organic compound. The intensity ranges from deep to light, as indicated, from red to white and from dark to light.

In contrast to the study of He Yangbo [4], we did not detect a large number of acids, and a small amount of propionic acid and butanoic acid was detected. Yang Jinjun et al. found that the volatile substances in different brands of red sour soup were different due to the differences in production technology and raw materials [5]. Xu Li et al. found that well-fermented sour soup contained more acids [32]. Similar to the results of this experiment, the relative percentage of the two acid substances in the foul-smelling samples decreased. Eight species of alkenes were detected; these compounds are generally believed to have originated from plants and carry a special plant aroma.

Sulfur compounds are produced by the enzyme methionine-demethanolase, which cleaves bonds between carbon and sulfur. These compounds are described as having a strong garlic taste and are generally considered to be unpleasant odours. However, at low concentrations, these compounds are considered to be ideal sources of aroma in wine [33]. 
Table 2. The relative percentage of volatile components in sour soup.

\begin{tabular}{|c|c|c|c|c|}
\hline \multirow{2}{*}{ Classification } & \multirow{2}{*}{ Volatile Component } & \multirow{2}{*}{ Statistical Significance } & \multicolumn{2}{|c|}{ Relative Content \% } \\
\hline & & & TF & STF \\
\hline \multirow[t]{25}{*}{ Esters } & Ethyl octanoate-M & * & $1.73 \pm 0.02$ & $1.32 \pm 0.05$ \\
\hline & Ethyl octanoate-D & * & $0.26 \pm 0.02$ & $0.16 \pm 0.03$ \\
\hline & Methyl salicylate & * & $0.50 \pm 0.00$ & $0.59 \pm 0.02$ \\
\hline & Propyl hexanoate-M & * & $3.58 \pm 0.07$ & $4.31 \pm 0.02$ \\
\hline & Propyl hexanoate-D & * & $1.64 \pm 0.08$ & $5.71 \pm 0.01$ \\
\hline & Ethyl heptanoate & * & $0.37 \pm 0.02$ & $0.56 \pm 0.01$ \\
\hline & Ethyl hexanoate-M & * & $3.96 \pm 0.03$ & $2.73 \pm 0.02$ \\
\hline & Ethyl hexanoate-D & * & $14.07 \pm 0.09$ & $16.43 \pm 0.02$ \\
\hline & Methyl hexanoate-M & * & $0.90 \pm 0.02$ & $0.64 \pm 0.00$ \\
\hline & Methyl hexanoate-D & * & $2.64 \pm 0.03$ & $3.14 \pm 0.01$ \\
\hline & Amyl acetate & * & $0.25 \pm 0.01$ & $0.32 \pm 0.00$ \\
\hline & Ethyl pentanoate-M & * & $1.20 \pm 0.00$ & $0.63 \pm 0.00$ \\
\hline & Ethyl pentanoate-D & * & $8.54 \pm 0.01$ & $9.97 \pm 0.00$ \\
\hline & Isoamyl acetate-M & * & $0.46 \pm 0.00$ & $0.28 \pm 0.00$ \\
\hline & Isoamyl acetate-D & * & $2.58 \pm 0.03$ & $2.74 \pm 0.00$ \\
\hline & Ethyl 3-methylbutanoate-M & * & $0.26 \pm 0.00$ & $0.22 \pm 0.00$ \\
\hline & Ethyl 3-methylbutanoate-D & * & $1.07 \pm 0.00$ & $1.78 \pm 0.00$ \\
\hline & Ethyl 2-hydroxypropanoate & * & $1.55 \pm 0.02$ & $1.36 \pm 0.00$ \\
\hline & Ethyl butanoate & * & $6.60 \pm 0.07$ & $5.55 \pm 0.03$ \\
\hline & Isobutyl acetate & * & $0.28 \pm 0.00$ & $0.40 \pm 0.00$ \\
\hline & Ethyl 2-methylpropanoate & * & $1.70 \pm 0.02$ & $1.49 \pm 0.02$ \\
\hline & Propyl acetate & & $2.64 \pm 0.03$ & $2.65 \pm 0.02$ \\
\hline & Ethyl propanoate & * & $2.46 \pm 0.01$ & $1.75 \pm 0.03$ \\
\hline & Ethyl acetate & * & $11.37 \pm 0.10$ & $9.37 \pm 0.10$ \\
\hline & Butyl acetate & * & $0.22 \pm 0.00$ & $0.24 \pm 0.00$ \\
\hline Pyrazines & Trimethyl pyrazine & & $1.36 \pm 0.00$ & $1.39 \pm 0.00$ \\
\hline \multirow{2}{*}{ Acids } & Propionic acid & * & $0.51 \pm 0.04$ & $0.17 \pm 0.00$ \\
\hline & Butanoic acid & * & $0.20 \pm 0.04$ & $0.10 \pm 0.00$ \\
\hline \multirow[t]{9}{*}{ Terpenes } & alpha-Pinene & * & $0.34 \pm 0.00$ & $0.16 \pm 0.00$ \\
\hline & Limonene & & $0.14 \pm 0.00$ & $0.14 \pm 0.00$ \\
\hline & 3-Carene & & $0.04 \pm 0.00$ & $0.03 \pm 0.00$ \\
\hline & alpha-Phellandrene & * & $0.02 \pm 0.00$ & $0.01 \pm 0.00$ \\
\hline & Myrcene & * & $0.07 \pm 0.00$ & $0.05 \pm 0.00$ \\
\hline & beta-Pinene & * & $0.04 \pm 0.00$ & $0.03 \pm 0.00$ \\
\hline & Thujene & $*$ & $0.08 \pm 0.00$ & $0.06 \pm 0.00$ \\
\hline & Terpinolene & * & $0.06 \pm 0.00$ & $0.07 \pm 0.00$ \\
\hline & Linalool & * & $0.08 \pm 0.00$ & $0.11 \pm 0.00$ \\
\hline \multirow[t]{7}{*}{ Alcohol } & 1-Hexanol & * & $0.17 \pm 0.00$ & $0.09 \pm 0.00$ \\
\hline & 2-Methylbutanol & * & $0.46 \pm 0.01$ & $0.48 \pm 0.00$ \\
\hline & 1-Propanol & * & $4.15 \pm 0.03$ & $3.27 \pm 0.07$ \\
\hline & Ethanol & * & $3.96 \pm 0.03$ & $3.00 \pm 0.06$ \\
\hline & (E)-2-hexenol-M & * & $0.03 \pm 0.00$ & $0.08 \pm 0.00$ \\
\hline & (E)-2-hexenol-D & * & $0.04 \pm 0.00$ & $0.22 \pm 0.00$ \\
\hline & 1-Pentanol & & $0.17 \pm 0.00$ & $0.17 \pm 0.00$ \\
\hline \multirow[t]{2}{*}{ Ketones } & 2-Butanone & * & $2.91 \pm 0.02$ & $1.91 \pm 0.07$ \\
\hline & acetone & * & $0.97 \pm 0.00$ & $0.47 \pm 0.00$ \\
\hline \multirow[t]{3}{*}{ Sulfocompounds } & Diallyl disulfide-M & & $0.11 \pm 0.01$ & $0.10 \pm 0.01$ \\
\hline & Diallyl disulfide-D & & $0.04 \pm 0.00$ & $0.03 \pm 0.01$ \\
\hline & Diallyl sulfide & $*$ & $0.13 \pm 0.00$ & $0.06 \pm 0.00$ \\
\hline Monoterpenes & 1,8-Cineole & * & $0.20 \pm 0.03$ & $0.50 \pm 0.01$ \\
\hline Aldehydes & 3-Methylbutanal & * & $0.49 \pm 0.00$ & $0.54 \pm 0.01$ \\
\hline
\end{tabular}

TF refers to the normal tomato fermentation sample; STF refers to the foul-smelling tomato fermentation sample. All analyses were conducted in triplicate, and the mean \pm SD is presented. M refers to monomer, and D refers to dimer. The * refers to the statistical significance difference between two groups samples. 


\subsection{Evaluation of Relative Odour Activity Value (ROAV) on Key Flavour Components of Sour Soup}

The relative aroma activity value (ROAV) was used to calculate the contribution value of volatile substances to flavour, and the main components of the flavour contribution of sour soup were further evaluated objectively. After compounds with ROAV values $<0.01$ were eliminated, the resulting ROAV value was used to obtain the heat map in Figure 6.
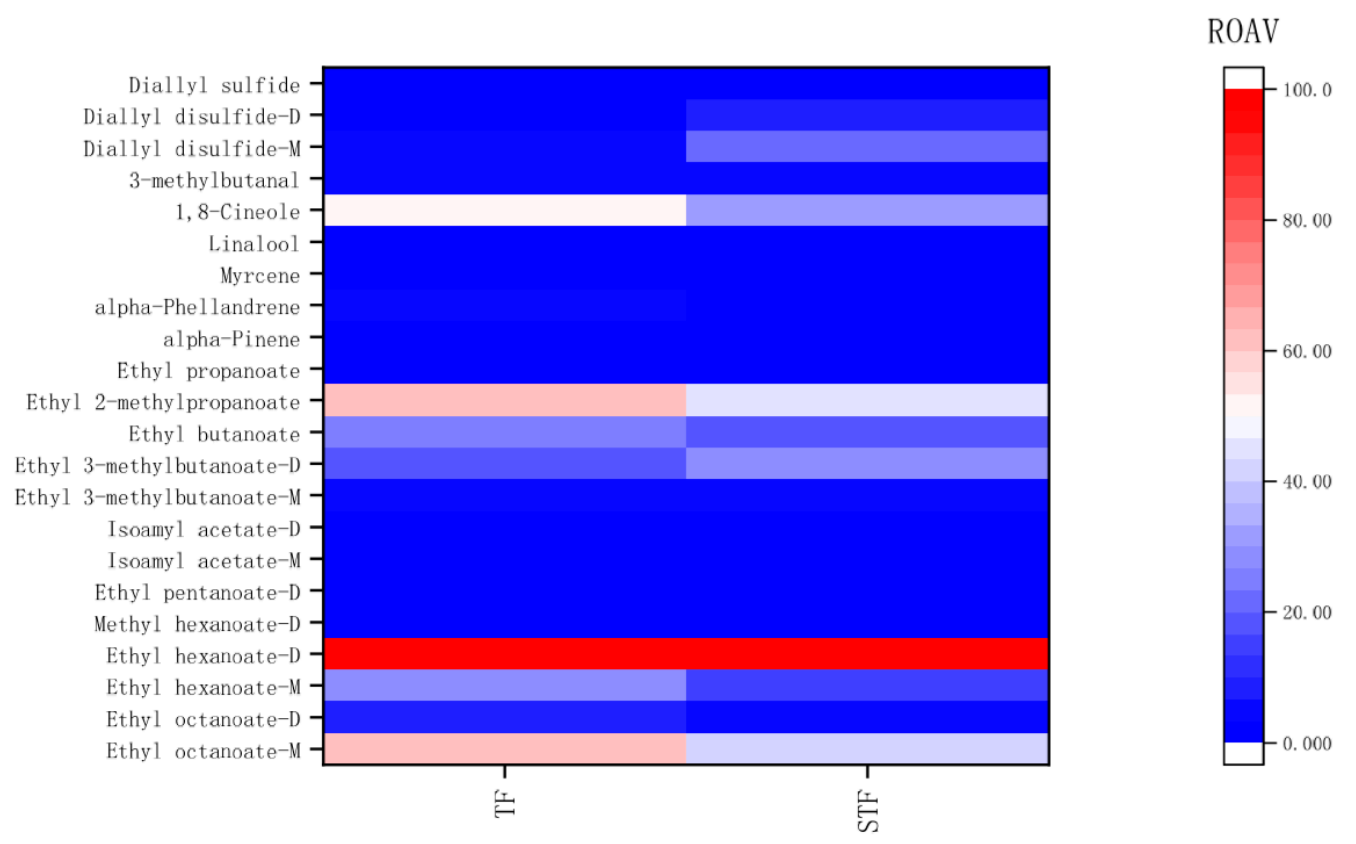

Figure 6. ROAV values of volatile components in sour soup. TF refers to the normal tomato fermentation sample; STF refers to the foul-smelling tomato fermentation sample. The colour code ranges from dark blue (lowest relative abundance) to dark red (highest relative abundance). The colour of each line represents the ROAV value of flavour, and each column represents a different studied sample in the heat map. Datapoints represent the means of three biological repeats. M refers to monomer, and D refers to dimer.

According to ROAV calculations, the volatile substances with ROAV > 1 were esters (ethyl octanoate-M, ethyl octanoate-D, ethyl hexanoate-M, ethyl hexanoate-D, isoamyl acetate-M, isoamyl acetate-D, ethyl 3-methylbutanoate-D, ethyl butanoate, isobutyl acetate), terpenoids (alpha-phellandrene, linalool, 1,8-cineole), aldehydes (3-methylbutanal), and sulfur-containing compounds (diallyl disulfide-M, diallyl disulfide-D), which play an important role in sour soup flavour. Volatile substances with $0.1<$ ROAV $<1$ included methyl hexanoate-D, ethyl pentanoate-D, isoamyl acetate-M, ethyl propanoate, alpha-pinene, myrcene, diallyl sulfide.

As shown in Figure 6, ethyl hexanoate-D is the dominant volatile substance in the sour soup; ethyl hexanoate-D has a sweet, fruity, and cucumber aroma [34]. Moreover, the ROAV values of 1,8-cineole, isobutyl acetate, ethyl butanoate, ethyl octanoate-M, and ethyl hexanoate-M of the normal samples were higher. After the peculiar smell was produced, the ROAV value of the foul-smelling samples decreased, and the ROAV values of diallyl disulfide-M and diallyl disulfide-D increased, which may be the main reason for the poor flavour of the foul-smelling samples.

\subsection{The Link Between Bacteria and the Flavour of Sour Soup}

Pearson correlation analysis was conducted for the bacteria (top 30) and the main volatile compounds (ROAV > 1), and the network visualization displayed by Cystoscope software is shown in Figure 7. According to Pearson correlation analysis, LAB are positively correlated with ester substances the same as other fermented vegetables [34], and secondly Pediococcus spp., 
Caproiciproducens spp., Clostridiumsensu_sensu_stricto_12 spp., and Oscillibacter were negatively correlated with ethyl octanoate-M, ethyl hexanoate-M, ethyl butanoate, and 2-methylpropanoate. Bacteroides spp., fibaculaceae_unclassified spp., Acinetobacter spp., and Halomonas spp. were negatively correlated with 1,8-cineole. There was a positive correlation between Bacteroides and diallyl disulfide-D. Pediococcus spp., Caproiciproducens spp., Clostridiumsensu_stricto_12 spp., Oscillibacter, Bacteroides spp., Fibaculaceae_unclassified spp., Acinetobacter spp. and Halomonas spp. may be the microbial factors that lead to flavour changes in sour soup.

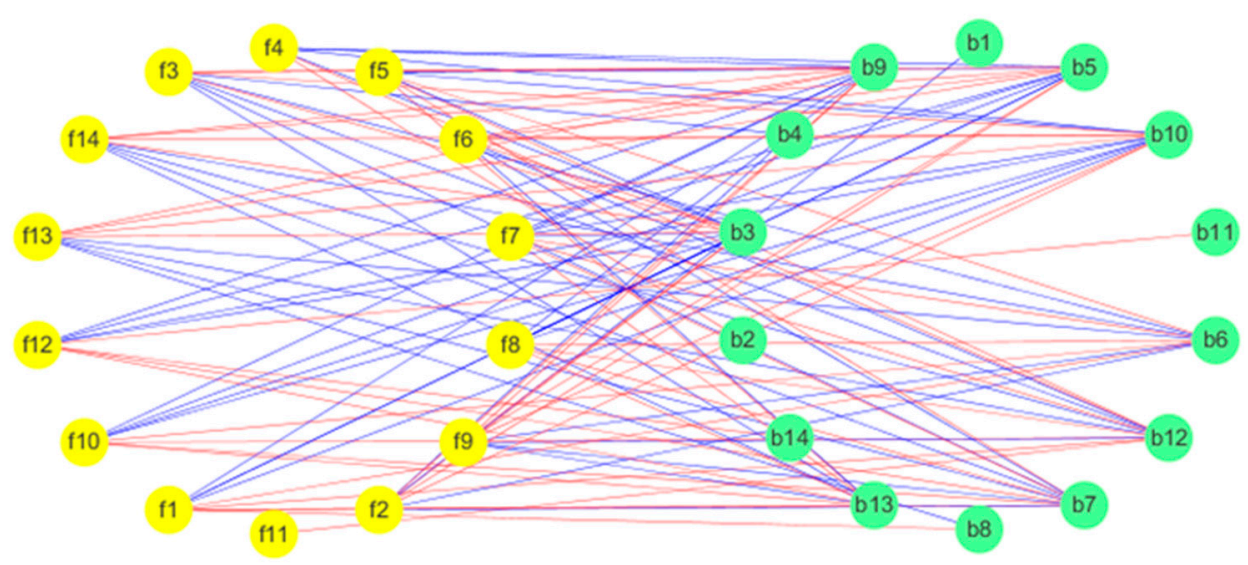

Figure 7. The correlation between microorganisms and volatile flavours. Yellow circles represent flavour components, and green circles represent bacterial genera. f1, ethyl octanoate-M; f2, linalool; $\mathrm{f} 3$, isoamyl acetate; $\mathrm{f} 4$, ethyl octanoate- $\mathrm{D}$; $\mathrm{f5}$, ethyl hexanoate-M; $\mathrm{f6}$, ethyl hexanoate- $\mathrm{D}$; $\mathrm{f}$, ethyl butanoate; f8, ethyl 3-methylbutanoate-M; f9, ethyl 3-methylbutanoate-D; f10, ethyl 2-methylpropanoate; f11, diallyl disulfide-D; f12, alpha-phellandrene; f13, 3-methylbutanal; f14, 1,8-cineole. b1, Bacteroides; b2, Ralstonia; b3, Pediococcus; b4, Parabacteroides; b5, Oscillibacter; b6, Halomonas; b7, f_Muribaculaceae_Unclassified; b8, Escherichia-Shigella; b9, Clostridium_sensu_stricto_12; b10, Caproiciproducens; b11, Bifidobacterium; b12, Bacteroides; b13, Acinetobacter.

\section{Conclusions}

In this paper, Illumina MiSeq sequencing and GC-IMS were used to investigate the role of the bacterial community in producing a peculiar smell in Chinese fermented sour soup. A total of 89 bacterial genera and 51 aroma compounds (including 25 esters, 8 terpenes, 8 alcohols, 3 sulfur compounds, 2 acids, 2 ketones, 1 pyrazine, 1 monoterpene and 1 aldehyde) were detected in the sour soup. Pediococcus spp., Caproiciproducens, Clostridiumsensu_stricto_12,Oscillibacter, Bacteroides spp., Fibaculaceae_unclassified spp., Acinetobacter spp., and Halomonas spp. were negatively correlated with ester and terpene compounds and positively correlated with sulfur-containing compounds, which may be the main cause of the flavour change in sour soup. This study provides microbial and metabolic information for optimizing the fermentation quality of sour soup and solving the odour problem.

Author Contributions: L.L.: Writing-original draft, Software, Visualization. J.W.: Formal analysis, Data curation. X.C.: Formal analysis, Data curation. L.H.: Data curation, Visualization. X.Z.: Formal analysis, Resources, Writing-review \& editing. X.G.: Conceptualization, Methodology, Project administration, Funding acquisition. All authors have read and agreed to the published version of the manuscript.

Funding: This research was funded by the Open Project Program of Guangdong Provincial Key Laboratory of Nutraceuticals and Functional Foods and the Program of Department of Natural Resources of Guangdong Province (GDNRC(2020)038).

Acknowledgments: Thanks to all the co-authors who participated in the work on this paper

Conflicts of Interest: The authors declare no conflict of interest. 


\section{References}

1. Huo, X.L. The Application and Development of Miao's Sour Diet Culture in Guizhou. J. Sichuan Tour. Univ. 2020, 1, 11-15.

2. Zhang, X. Study on the Microbial Flora of Semi-Finished Products of "Red Sour Soup" in Guizhou. Master's Thesis, Southwest University, Chongqing, China, 2011.

3. Zeng, R.M.; Han, L.; Huang, P. Research Progress and Industrialization Process on Guizhou Red Sour Soup Hotpot Seasoning. Food Ferment. Technol. 2014, 50, 72-75.

4. Yang-bo, H.; Yong-fu, L.; Ding-jiang, Z. Analysis on the Flavor Difference of Red Sour Soup Including Urn Odor and Normal Flavor Samples with Electronic Nose and Gas Chromatography-Ion Mobility Spectroscopy. Sci. Technol. Food Ind. 2020, 137, 109439.

5. Yang., J.J.; Hu, J.X. Analysis of the volatile flavor substances in different red sour soup based on electronic nose and GC-MS. Food Ferment. Ind. 2020, 46, 234-242. [CrossRef]

6. Wang, Q.Q.; Tian, J.X.; Pang, Z.D.; Du, J. Analysis of dominant microflora associated with the unique flavor of Guizhou Kaili sour Soup using Illumina MiSeq sequencing. Food Ferment. Ind. 2020, 46, 40-47. [CrossRef]

7. Liu, Z.; Li, J.; Wei, B.; Huang, T.; Xiao, Y.; Peng, Z.; Xie, M.; Xiong, T. Bacterial community and composition in Jiang-shui and Suan-cai revealed by high-throughput sequencing of 16S rRNA. Int. J. Food Microbiol. 2019, 306, 108271. [CrossRef]

8. Wang, S.; Chen, H.; Sun, B. Recent progress in food flavor analysis using gas chromatography ion mobility spectrometry (GC-IMS). Food Chem. 2020, 315, 126158. [CrossRef]

9. Li, X.; Cui, W.; Wang, W.; Wang, Y.; Gong, Z.; Xu, Z. Analysis of the volatile compounds associated with pickling of ginger using headspace gas chromatography ion mobility spectrometry. Flavour Fragr. J. 2019, 34, 485-492. [CrossRef]

10. Liang, H.; Chen, H.; Zhang, W.; Yu, C.; Ji, C.; Lin, X. Investigation on microbial diversity of industrial Zhacai paocai during fermentation using high-throughput sequencing and their functional characterization. LWT 2018, 91, 460-466. [CrossRef]

11. Lin, L.-J.; Du, F.-M.; Zeng, J.; Liang, Z.-J.; Zhang, X.-Y.; Gao, X.-Y. Deep insights into fungal diversity in traditional Chinese sour soup by Illumina Miseq Sequencing. Food Res. Int. 2020, 137, 109439. [CrossRef]

12. Du, F.; Zhang, X.; Gu, H.; Song, J.; Gao, X. Dynamic Changes in the Bacterial Community During the Fermentation of Traditional Chinese Fish Sauce (TCFS) and their Correlation with TCFS Quality. Microorganisms 2019, 7, 371. [CrossRef] [PubMed]

13. Gresse, R.; Durand, F.C.; Dunière, L.; Blanquet-Diot, S.; Damiano-Forano, E.; Durand, C.; Blanquet-Diot, S. Microbiota Composition and Functional Profiling Throughout the Gastrointestinal Tract of Commercial Weaning Piglets. Microorganisms 2019, 7, 343. [CrossRef] [PubMed]

14. Rodríguez López, J.; Grande, M.J.; Pérez-Pulido, R.; Galvez, A.; Lucas, R. Impact of High-Hydrostatic Pressure Treatments Applied Singly or in Combination with Moderate Heat on the Microbial Load, Antimicrobial Resistance, and Bacterial Diversity of Guacamole. Microorganisms 2020, 8, 909. [CrossRef] [PubMed]

15. Deng, Z.-S.; Zhang, B.-C.; Qi, X.-Y.; Sun, Z.-H.; He, X.-L.; Liu, Y.-Z.; Li, J.; Chen, K.-K.; Lin, Z.-X. Root-Associated Endophytic Bacterial Community Composition of Pennisetum Sinese from Four Representative Provinces in China. Microorganisms 2019, 7, 47. [CrossRef] [PubMed]

16. Fang, G.Y.; Huang, B.W.; Jiang, Y.J. Dynamic Succession of Bacterial Community Structure during the Fermentation Process of Zhejiang Rosy Vinegar Analyzed by High Throughput Sequencing. Food Sci. 2020, 41, 125-133. [CrossRef]

17. Xiao, Y.; Xiong, T.; Peng, Z.; Liu, C.; Huang, T.; Yu, H.; Xie, M. Correlation between microbiota and flavours in fermentation of Chinese Sichuan Paocai. Food Res. Int. 2018, 114, 123-132. [CrossRef]

18. Xu, X.; Wu, B.; Zhao, W.; Pang, X.; Lao, F.; Liao, X.; Wu, J. Correlation between autochthonous microbial communities and key odorants during the fermentation of red pepper (Capsicum annuum L.). Food Microbiol. 2020, 91, 103510. [CrossRef]

19. Liang, H.; He, Z.; Wang, X.; Song, G.; Chen, H.; Lin, X.; Ji, C.; Li, S. Effects of salt concentration on microbial diversity and volatile compounds during suancai fermentation. Food Microbiol. 2020, 91, 103537. [CrossRef]

20. Ye, L.; Li, Y.; Wang, R.R.; Liu, C.G.; Jiang, L.W.; Deng, F.M.; Zhou, H. Progress in Research on the Diversity of Lactic Acid Bacteria in Traditional Chinese Fermented Vegetables. Food Sci. 2018, 39, 296-301. 
21. Byung-Chun, K.; Byoung, S.J.; Seil, K.; Hyunook, K.; Youngsoon, U.; Byoung-In, S. Caproiciproducens galactitolivorans gen. nov., sp. nov., a bacterium capable of producing caproic acid from galactitol, isolated from a wastewater treatment plant. Int. J. Syst. Evol. Microbiol. 2015, 65, 4902-4908.

22. Liu, T.T.; Ma, Y.S.; Li, N.; Zhao, X. Comparison of Aroma Characteristics of Cerasus humilis Wines Fermented from Whole Fruits and Pomace. Food Sci. 2016, 37, 99-104. [CrossRef]

23. Zhang, H.; Meng, Y.; Wang, Y.; Zhou, Q.; Li, A.; Liu, G.; Li, J.; Xing, X. Prokaryotic communities in multidimensional bottom-pit-mud from old and young pits used for the production of Chinese Strong-Flavor Baijiu. Food Chem. 2020, 312, 126084. [CrossRef] [PubMed]

24. Huang, D.; Liu, Y.Q.; Ni, Y. Study of Main Aroma Components of Sichuan Bran Vinegar Based on ROAV Value. Food Ind. 2016, 37, 288-292.

25. Zhang, D.Y.; Xu, L.; Lu, Q.S.; Mou, Q. Optimization of Kaili red sour soup tomato juice fermentation process and fortified microflora. Food Sci. Technol. 2018, 43, 313-318. [CrossRef]

26. Zheng, S.S.; Hu, P. Study on Quality Change of Red Sour Soup Fermented by Lactic Acid Bacteria. China Condiment 2019, 44, 65-70. [CrossRef]

27. Dzialo, M.C.; Park, R.; Steensels, J.; Lievens, B.; Verstrepen, K.J. Physiology, ecology and industrial applications of aroma formation in yeast. FEMS Microbiol. Rev. 2017, 41 (Suppl. S1), S95-S128. [CrossRef]

28. Hayaloglu, A.A.; Cakmakci, S.; Brechany, E.Y.; Deegan, K.C.; McSweeney, P.L.H. Microbiology, biochemistry, and volatile composition of Tulum cheese ripened in goat's skin or plastic bags. J. Dairy Sci. 2007, 90, 1102-1121. [CrossRef]

29. Ko, A.Y.; Rahman, M.M.; Abd El-Aty, A.M.; Jang, J.; Choi, J.H.; Mamun, M.I.R.; Shim, J.H. Identification of volatile organic compounds generated from healthy and infected powdered chili using solvent-free solid injection coupled with GC/MS: Application to adulteration. Food Chem. 2014, 156, 326-332. [CrossRef]

30. Rosita, G.; Hammond, E.G. Generation of Swiss Cheese Flavor Components by the Reaction of Amino Acids with Carbonyl Compounds. J. Dairy Sci. 1989, 72, 604-613.

31. Mestres, M.; Busto, O.; Guasch, J. Analysis of organic sulfur compounds in wine aroma. China Condiment 2000, 44, 76-80. [CrossRef]

32. Xu, L.; Dai, Y.Z. Effects of Different Lactic Acid Bacteria in Sour Soup on Volatile Flavor Compounds. Food Sci. 2008, 29, 505-509. [CrossRef]

33. Xiao, Z.B.; Zhou, X.; Niu, Y.W.; Zhao, F.F. The Analysis of the Characteristic Aroma Components of Cherry Wine by Gas Chromatography-Olfactometry (GC-O) and Odor Activity Values (OAV). J. Chin. Inst. Food Sci. Technol. 2017, 17, 246-254. [CrossRef]

34. Rao, Y.; Tao, Y.; Chen, X.; She, X.; Qian, Y.; Li, Y.; Du, Y.; Xiang, W.; Li, H.; Liu, L.; et al. The characteristics and correlation of the microbial communities and flavors in traditionally pickled radishes. LWT 2020, 118, 108804. [CrossRef]

(C) 2020 by the authors. Licensee MDPI, Basel, Switzerland. This article is an open access article distributed under the terms and conditions of the Creative Commons Attribution (CC BY) license (http://creativecommons.org/licenses/by/4.0/). 